Esta revista forma parte del acervo de la Biblioteca Jurídica Virtual del Instituto de Investigaciones Jurídicas de la UNAM

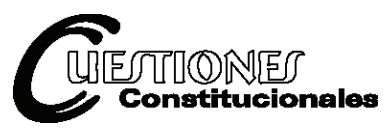

Revista Mexicana de Derecho Constitucional

Núm. 36, enero-junio 2017

\title{
LA REFORMA POLÍTICA DE LA CIUDAD DE MÉXICO*
}

\author{
THE POLITICAL REFORM IN MEXICO CITY
}

\author{
Enrique RABELL GARCÍA**
}

RESUMEN: La creación de la Asamblea de Representantes en 1987 de la Ciudad de México, así como la alternancia política, han motivado que la reforma política del Distrito Federal sea una constante. Las propuestas van desde su transformación en otra entidad federativa de la República hasta la creación de una jurisdicción con autonomía constitucional. El presente estudio tiene por hipótesis principal señalar que una estructura autonómica otorga mayores privilegios al gobierno de la Ciudad de México en comparación con las entidades federativas. En cuanto a las competencias y facultades de los poderes de la Ciudad y de las delegaciones, éstas dependerán en primer término del ámbito mínimo de competencias que debe conservar la Federación y, en segundo término, aquellas de las autoridades citadinas frente a las delegacionales.

Palabras clave: derecho constitucional, políticas públicas, administración pública, autonomía.
ABSTRACT: The political reform in Mexico City, as Federal District, has been an ongoing issue since the foundation of the Assembly of Representatives in 1987, as well as the political turnovers within government. The political proposals comprehend the creation of another federal state government to the creation of a new constitutional autonomous government. The study establishes as main hypothesis that a city government with autonomy provides more privileges in front of the federal state governments. In regard to the attributions of the City government, these would depend first in the minimal responsibilities kept for the $\mathrm{Fe}$ deral government and, in a second stage, the attributions divided between the City central government and the rest of the local governments.

Descriptors: Constitutional Law, Public Policy, Public Administration, Autonomy.

* Artículo enviado por la Revista General de Derecho Constitucional como parte de las celebraciones por los 100 años de la Constitución.

** Profesor de tiempo completo en la Facultad de Derecho, UAQ, erabell@mail.com. 
Esta revista forma parte del acervo de la Biblioteca Jurídica Virtual del Instituto de Investigaciones Jurídicas de la UNAM

SUMARIO: I. Introducción. II. Marco teórico. III. Antecedentes históricos. IV. Instituciones vigentes. V. Desarrollo urbano. VI. Propuesta de reforma política. VII. Conclusiones. VIII. Bibliografia.

\section{INTRODUCCIÓN}

El gobierno de la Ciudad de México, como capital de la República, ha sido motivo de importantes controversias por su naturaleza jurídica desde la independencia nacional. La fórmula original consistió en ser considerada un departamento central del sector federal. Hoy en día, a raíz de la última reforma constitucional de 2016, cuenta con un régimen constitucional único en el cual se confunden las figuras o normas que aplican a los estados de la República y los principios para darle plena autonomía.

La capital del país en un sistema federal no solamente es el ámbito jurídico de los poderes federales, sino también el ámbito territorial donde se garantiza que los poderes de la unión tengan independencia y seguridad para poder desarrollar sus tareas. De ahí que la fórmula federalista original consideraba a la ciudad capital como un territorio centralizado de los poderes de la unión. Esto es, las autoridades locales o de la capital eran designados por la autoridad federal y actuaban en representación de tales poderes federales. Al paso del tiempo, y el crecimiento de las grandes ciudades capitales, varias fórmulas se han aplicado para otorgar mayor legitimidad a las autoridades locales de la capital, así como darles a sus habitantes una participación política efectiva. Hoy en día, entre los extremos de continuar como un departamento central o ser una entidad con soberanía interior, se optó por un estatuto con competencias propias para la Ciudad de México.

La reforma política actual propone para ciertos sectores que se elimine dicho estatuto y se convierta en una verdadera entidad federativa, con el supuesto de que dicha reforma le dará más poder, competencias, legitimidad y estatus político a la propia Ciudad de México. Las interrogantes principales de esta postura consisten en: ¿Qué pasará con el ámbito territorial de los poderes de la Unión? ¿Ganarán o perderán más competencias en este nuevo arreglo? ¿Quién tendrá el mando de seguridad? ¿Cuál sería el arreglo fiscal? En este orden de ideas se plantea como hipótesis de trabajo que en virtud de las actuales reglas del federalismo, las cuales favorecen a los poderes de la Unión y no a las entidades — casos con mayor equilibrio sucede 
en el federalismo de Estados Unidos, Canadá o Alemania, por ejemplo-convertir a la Ciudad de México en estado federal le restaría más competencias y responsabilidades. Siendo la hipótesis alternativa en el sentido de ampliar su estatuto de autonomía para otorgar mayores competencias al gobierno de la Ciudad capital.

Con la finalidad de analizar esta problemática se plantea utilizar diversos enfoques, entre ellos el histórico, constitucional, financiero y administrativa, para en forma conjunta desarrollar una interpretación integral y aportar conclusiones que sirvan al análisis constitucional del régimen jurídico de la Ciudad de México. En términos generales se establecen como partes generales del estudio la metodología, el análisis y la discusión final o resultados. La parte metodológica, entendida ésta como la relativa a la descripción de la problemática, comprende diversos apartados. El primero corresponde al marco teórico sobre los conceptos de federalismo y autonomía con la finalidad de conocer los alcances y límites de estas formas de Estado. El segundo comprende el desarrollo histórico del gobierno de la Ciudad de México desde la Constitución federalista de 1824 a la fecha. Además se complementa con dos apartados que comprenden el apartado a las instituciones vigentes, que abarca el régimen constitucional y el fiscal; seguido de otro apartado sobre la materia de desarrollo urbano. Los apartados anteriores inician la discusión sobre las consecuencias de transitar hacia un régimen de entidad federativa o de autonomía. El análisis se presenta en la sección sobre la propuesta de reforma política. Se estudia en particular el nombre oficial, el caso de la Constitución local, las facultades del Ejecutivo y Legislativo de la Ciudad, así como las competencias de los gobiernos locales de la capital. La discusión final se presenta en el último apartado de conclusiones, en el cual se consideran los aspectos positivos y negativos de la reforma política y constitucional reciente.

\section{MARCO TEÓRICO}

\section{Federación}

El sistema federativo actual es el resultado de la inestabilidad política de la Confederación Americana de 1777 y la necesidad de contar con una defensa militar común frente a Inglaterra y otras potencias europeas. En el aspecto doctrinal la federación busca conciliar el interés por tener un órgano 
Esta revista forma parte del acervo de la Biblioteca Jurídica Virtual del Instituto de Investigaciones Jurídicas de la UNAM

con fuerza política y legal para aplicar directamente sus disposiciones y, por el otro, conservar el máximo grado de autonomía de los estados miembros de la unión federal.

Históricamente, Ferrando señala que

se distinguen dos modos fundamentales de formación de los Estados federales: puede ser el resultado de la transformación de un Estado unitario en federal, en cuyo caso - como observa biscaretti di ruffía - se produce realmente el nacimiento de los Estados-miembros y no del Estado federal, que es el antiguo Estado unitario constitucionalmente transformado: Brasil en 1891, Austria en 1920, Méjico y la U.R.S.S. se formaron según este procedimiento. El segundo modo, que es el normal, tiene lugar cuando se reúnen varios Estados, hasta entonces independientes, originando un nuevo Estado federal. Tal es, por ejemplo, el caso de los Estados Unidos, de Suiza, de Argentina, de la Confederación de Alemania del Norte, etcétera. Este proceso puede realizarse jurídicamente, a través de un tratado internacional primero y de una Constitución después (Estados Unidos), o bien por vía extrajurídica o de hecho (Suiza). ${ }^{1}$

Las características principales de un Estado federal consisten en la creación de una nueva entidad política o Federación que representa a todas las entidades, la participación de las entidades federativas en el gobierno nacional y el reparto de facultades entre la Federación y sus entidades. Tena Ramírez comenta que "en la federación los Estados-miembros pierden totalmente su soberanía exterior y ciertas facultades interiores en favor del gobierno central, pero conservan para su gobierno propio las facultades no otorgadas al gobierno central". ${ }^{2}$ Por tanto, las características principales del estado federal son:

a) La creación de un nuevo estado a partir de naciones independientes.

b) El reparto de competencias entre la federación y las entidades, establecidas en la Constitución nacional.

1 Ferrando Badía, Juan, El Estado unitario, el federal y el Estado autonómico, 2a. ed., Madrid, Tecnos, 1986, p. 79.

2 Tena Ramírez, Felipe, Derecho constitucional mexicano, 22a. ed., México, Porrúa, 1987, p. 113. 
Esta revista forma parte del acervo de la Biblioteca Jurídica Virtual del Instituto de Investigaciones Jurídicas de la UNAM

c) La federación representa la unidad nacional, cuenta con la soberanía exterior y materias exclusivas de ámbito nacional para asegurar la unidad interior.

d) Las entidades conservan su soberanía interior, por tanto sus autoridades son electas en la propia entidad y cuentan con su propia Constitución dentro de los límites de la Constitución general.

e) Las entidades participan en el gobierno nacional por medio de la representación en el cuerpo legislativo nacional y en el proceso de reforma constitucional de la unión.

\section{Regionales o autonómicos}

\section{González Casanova (1989: 334-335) comenta que}

el origen contemporáneo de las comunidades autónomas se encuentra en la necesidad de los Estados unitarios de reconocer ciertos poderes de autogobierno a grupos de población caracterizados por su unidad nacional-cultural y lingüística o por su condición insular o de alejamiento geográfico del territorio físico del Estado. Tras la I Guerra Mundial, Inglaterra, Checoslovaquia, Finlandia y España otorgaron estatutos de autonomía respectivamente a Irlanda del Norte, Rusia subcarpática, Islas Aaland, Cataluña y País Vasco. ${ }^{3}$

El Estado autonómico tiene por característica que el poder central conserva la soberanía y reparte atribuciones de acuerdo con las situaciones particulares de cada región. De esta forma, cada región podrá tener diferentes materias y competencias públicas en relación con las demás regiones de la misma nación. Respecto de esta forma de Estado, González Casanova señala como distinciones respecto del Estado federal:

La potestad autoconstituyente del Estado federado es originaria, pues fue Estado antes que el federal. En cambio, la comunidad autónoma es derivada del poder central. Las entidades federativas sólo se hayan sometidas a los controles jurídicos estrictamente fijados en la Constitución general. En el Estado unitario ha de dejar sin concretar en el texto constitucional aquellos controles concretos. Las entidades federativas participan con paridad en la

3 González Casanova, J. A., Teoría del Estado y derecho constitucional, 3a. ed., Barcelona, Vicens-Vives, 1989, pp. 334 y 335. 
Esta revista forma parte del acervo de la Biblioteca Jurídica Virtual del Instituto de Investigaciones Jurídicas de la UNAM

reforma constitucional y en la elaboración de leyes federales, mediante una segunda cámara en el Congreso. En el Estado unitario, las comunidades autónomas carecen de esta facultad. ${ }^{4}$

El estado autonómico o regional se crea en Italia y España, como forma de rechazo al sistema unitario y, también, como negativa al federalismo que otorga un mismo trato a todas las entidades. El estado autonómico nace para conceder estatutos especiales a favor de ciertas regiones; en Italia es el caso de Toscana y Umbría, y en España para favorecer a las regiones de Cataluña y el País Vasco. ${ }^{5}$

En España la autonomía nace efectivamente a partir de la Constitución de 1931 al establecerse el Estado integral. ${ }^{6}$ Por lo cual fue necesario encontrar el sentido que dieron las Cortes Constituyentes al nuevo concepto político. Ferrando Badía señala que Jiménez de Asúa, presidente de la Comisión de Constitución, deliberó:

Frente al Estado unitario, tiene el integral la ventaja, en nuestro caso, de ser compatible, sin imponerlas, con diversos grados de autonomías regionales, cuando sean pedidas y procedentes, junto a un régimen de vinculación de otros territorios nacionales no preparados para aquellas formas de autarquía. $\mathrm{Y}$ frente al Estado federal tiene el provecho de permitir, sin desnaturalizarse, la existencia de estos territorios, ligados por estrecha dependencia políticoadministrativa al Estado... junto a aquellas otras regiones que quieran y estén capacitadas para asumir funciones de autodeterminación, en grado de distinta intensidad, que son variantes de matiz en las posibles autonomías regionales diversas, sin importar una relación uniforme entre el Estado y unos y otros territorios.

Aunque dicha Constitución no tuvo aplicación plena, debido a la Guerra Civil, sirvió como fundamento e inspiración de la actual Constitución española de $1978 .^{7}$

4 Ibidem, p. 37.

5 Ferrando Badía, Juan, El Estado unitario..., cit., p. 171.

6 Párrafo tercero, artículo 1o. "La República constituye un Estado integral, compatible con la autonomía de los municipios y las regiones...”. Párrafo primero, artículo 80. "El Estado español, dentro de los límites irreductibles de su territorio actual, estará integrado por municipios mancomunados en provincias y por las regiones que se constituyan en régimen de autonomía".

7 Ferrando Badía, Juan, El Estado unitario..., cit., p. 171. 
Esta revista forma parte del acervo de la Biblioteca Jurídica Virtual del Instituto de Investigaciones Jurídicas de la UNAM

Concluyendo, las principales características de un Estado regional o autónomo son:

a) La existencia de un ordenamiento constitucional, como único titular de soberanía y sólo un poder constituyente.

b) Las entidades autónomas carecen de la facultad de otorgarse su propio estatuto o constitución local, sino que le es impuesto por el órgano legislativo central.

c) No existe una división clara de facultades entre el gobierno central y las comunidades autónomas, sino que cada comunidad autónoma puede gozar de facultades propias y distintas a las demás comunidades; esto de acuerdo al estatuto promulgado por las cortes generales o centrales.

d) Las entidades autónomas no participan del proceso de reforma constitucional nacional.

\section{ANTECEDENTES HISTÓRICOS}

En razón de la época prehispánica, Salomón sintetiza que

la Ciudad de México ha sido, desde los tiempos prehispánicos, el centro político y económico más importante del país. Los aztecas no sólo la fundaron, también le dieron la grandeza que maravilló al conquistador en 1521 . Arrasada por Hernán Cortés, la antigua Tenochtitlán habría de transformarse en la capital de la Nueva España. Durante el coloniaje español, la ciudad de México adquirió una nueva dimensión política y cultural y económica que la convirtieron en la Nobilísima Ciudad Metropolitana y Cabeza del Reino. La lucha por la independencia cambió el destino político del país y el de la Ciudad de México. ${ }^{8}$

Ya en el siglo XIX, la Ciudad de México es reconocida como asiento de los poderes en las Constituciones federales de 1824, restauración de vigencia en 1846, la de 1857 y con el triunfo de la República en 1867. Y, como contrapartida, las Constituciones centralistas no cuentan con distrito

8 Salomón Díaz, Alfaro, "El Distrito Federal mexicano. Breve historia constitucional", Estudios jurídicos en torno a la Constitución mexicana de 1917 en su septuagésimo quinto aniversario, México, UNAM, 1992, p. 199. 
Esta revista forma parte del acervo de la Biblioteca Jurídica Virtual del Instituto de Investigaciones Jurídicas de la UNAM

federal por suprimirse la Federación. ${ }^{9}$ En cuanto a su organización, la Ciudad de México se organizaba en municipios gobernados por ayuntamientos. En este caso las Constituciones federales no incluyeron al municipio, por considerarlo una entidad dentro de la soberanía de los estados. Y, en contrapartida, las Constituciones centralistas sí lo incluían como la base de la organización de los departamentos. Sin embargo, aun en los esquemas federales la Ciudad de México continuó dividiéndose en municipios por medio de leyes secundarias.

Tena Ramírez señala que el Distrito Federal se creó como consecuencia del artículo 50, fracciones XXVIII y XXIX, de la Constitución federal de 1824, que facultaba al Congreso para "elegir un lugar que sirva de residencia a los supremos poderes de la Federación y ejercer en su distrito las atribuciones del Poder Legislativo de un Estado...". ${ }^{10}$ En consecuencia, el Congreso Constituyente expidió el decreto de 18 de noviembre de 1824 por el cual se estableció que la residencia de los supremos poderes de la Federación, es decir, el Distrito Federal, sería la Ciudad de México, en un radio de dos leguas cuyo centro sería la plaza mayor de la ciudad. Aunque no se mencionaba en la carta federal, se nombró a un gobernador de la capital y se conservó la figura municipal anexando poblaciones vecinas. ${ }^{11}$

Posteriormente, Antonio López de Santa Anna, con el decreto del 16 de febrero de 1854, ensanchó la extensión del Distrito Federal y estableció la división en ocho prefecturas interiores y tres prefecturas exteriores. ${ }^{12}$ Por su

9 Instituto Nacional para el Federalismo y el Desarrollo Municipal, México, Secretaría de Gobernación, 2013, disponible en: http://www.inafed.gob.mx/work/enciclopedia/ EMM09DF/historia.html

10 Tena Ramírez, Felipe, Leyes fundamentales de México, México, Porrúa, 1985, p. 29.

11 La porción norte de la entonces Villa de Guadalupe Hidalgo, terrenos de la hacienda de Santa Ana Aragón, pueblo del Peñón de los Baños y Ticomán. Por el oriente: la hacienda del Peñón de los Baños, terrenos de la hacienda de los Reyes, pueblo de Santa Martha Acatitla y parte poniente de Ixtapalapa. Por el sur: Churubusco, parte norte de Coyoacán, pueblo de Axotla y terrenos de la hacienda de San Borja. Por el poniente: Santa María Nonoalco, zona en donde se ubica actualmente la colonia San Pedro de los Pinos, poniente de Tacubaya, Chapultepec y Tacuba, así como una porción territorial de la actual delegación Azcapotzalco. Dublán, Manuel y José María Lozano, Legislación mexicana, México, Imprenta del Comercio, t. VII, 1876, pp. 49-51.

12 “... al norte, hasta el pueblo de San Cristóbal Ecatepec; al N.O., Tlanepantla; al poniente los Remedios, San Bartolo y Santa Fe; al S.O., desde el límite oriente de Huisquilucan, Mixcoac, San Ángel y Coyoacán; por el sur, Tlalpan; por el S.E., Tepepan, Xochimilco e Iztapalapa; por el O., el Peñón Viejo y entre este rumbo y el N. E. y N., hasta la medianía de las aguas del lago de Texcoco". Idem. 
Esta revista forma parte del acervo de la Biblioteca Jurídica Virtual del Instituto de Investigaciones Jurídicas de la UNAM

parte, la Constitución de 1857 introduce el concepto del Estado del Valle de México, sólo en caso de que los poderes federales se trasladasen a otro lugar, contemplado esta situación en el artículo 46 y el artículo 72, fracciones $\mathrm{V}$ y VI que facultaban al Congreso para cambiar la residencia de los poderes federales y arreglo interior del Distrito Federal. ${ }^{13}$

Por decreto del 6 de mayo de1861 se dio otra reforma administrativa al Distrito Federal, quedando en Municipalidad de México y cuatro partidos, siendo los de Guadalupe, Xochimilco, Tlalpan y Tacubaya. Esta división política del Distrito Federal se mantuvo hasta diciembre de 1899, cuando bajo el gobierno de Porfirio Díaz se agregaron cuatro municipios más (Cuajimalpa, Tlaltenco, Mixtic y San Pablo Ostotepec) y se amplió de cuatro a seis partidos o distritos, agregando las de Azcapotzalco y Coyoacán.

En 1903, la Ley de Organización Política y Municipal del Distrito Federal, dividió el territorio en 13 municipalidades. ${ }^{14}$ La Constitución de 1917 ratifica a la Ciudad de México como residencia de los poderes federales y, por medio de la Ley de Organización del Distrito y Territorios Federales del 14 de abril de ese año, se confirman las trece municipalidades existentes. Dicha división fue modificada al crearse la municipalidad General Anaya en 1924.

El texto original de la Constitución vigente ya incluía al Distrito Federal como parte integrante de la Federación. Además, la fracción VI del artículo 73 establecía el régimen municipal del Distrito Federal y territorios; el reconocimiento de un ayuntamiento para gobernar cada municipio; el Distrito Federal y territorios estaría a cargo de un gobernador que dependería del presidente de la República; la elección de magistrados y jueces nombrados por el Congreso; y un Ministerio Público a cargo de un procurador general que sería nombrado y removido por el presidente. ${ }^{15}$

Ante la iniciativa del presidente Obregón en mayo de 1928, la cual mencionaba las dificultades políticas y administrativas de los municipios y el

13 Salomón Díaz, Alfaro, "El Distrito Federal..., cit., p. 203.

14 Las municipalidades serían: México, Guadalupe Hidalgo, Azcapotzalco, Tacuba, Tacubaya, Mixcoac, Cuajimalpa, San Ángel, Coyoacán, Tlalpan, Xochimilco, Milpa Alta e Iztapalapa. Dublán, Manuel y Lozano, José María, Legislación mexicana, México, Imprenta del Comercio, 1876, t. XXXV, pp. 336-357.

15 Jara se pronunció por que el municipio de la Ciudad de México tuviera todas las prerrogativas del resto de los de la República, librándose de la acción del gobernador del Distrito. En: Instituto Nacional de Estudios Históricos de la Revolución Mexicana, Congreso Constituyente 1916 a 1917, Diario de Debates, México, INEHRM, 1985, t. II, pp. 364 y 365 . 
Esta revista forma parte del acervo de la Biblioteca Jurídica Virtual del Instituto de Investigaciones Jurídicas de la UNAM

predominio de los poderes federales sobre los servicios locales, se suprime en el Distrito Federal el municipio libre y se encarga su gobierno al presidente de la República, quien lo ejercería por conducto del órgano u órganos que determinara la ley respectiva. De esta forma, en diciembre de 1928, ya siendo presidente Portes Gil, se publica la Ley Orgánica del Distrito y de los Territorios Federales, en la cual queda el presidente como encargado de las funciones administrativas por medio de un Departamento del Distrito Federal, creándose al efecto 13 delegaciones. ${ }^{16}$

La Ley Orgánica del Departamento del Distrito Federal del 31 de diciembre de 1941, crea como ente jurídico a la Ciudad de México (lo que fue sede del Departamento Central y delegación General Anaya) y 12 delegaciones. En 1970 se da otra reforma para dividir el territorio, quedando lo que fue la jurisdicción de la Ciudad de México en cuatro delegaciones. ${ }^{17}$

De gran trascendencia política fue la creación de la Asamblea de Representantes del Distrito Federal mediante reforma constitucional al artículo 73, fracción VI, de fecha 10 de agosto de 1987. La Asamblea se funda como un órgano de representación ciudadana, integrado en ese momento por 40 representantes electos por mayoritaria relativa y 26 por representación proporcional. Se le dio competencia para dictar bandos, ordenanzas y reglamentos de policía y buen gobierno en materia de servicios; aprobar nombramientos de magistrados del Tribunal Superior de Justicia e iniciar ante el Congreso de la Unión leyes o decretos en materia relativa al Distrito Federal. ${ }^{18}$

Con dicho antecedente, se procedió el 25 de octubre de 1993 a la creación de una nueva figura constitucional que contempla la actual naturaleza jurídica y organización del Distrito Federal al modificar los artículos 31, 44, 73, 74, 79, 89, 104, 105, 107 y 122, así como la denominación del título V, adición de una fracción IX al artículo 76 y un primer párrafo al 199; derogándose al efecto la fracción XVII del artículo 89 de la propia Constitución de la República. ${ }^{19}$

16 Son: Guadalupe Hidalgo, Azcapotzalco, Ixtacalco, General Anaya, Coyoacán, San Ángel, La Magdalena Contreras, Cuajimalpa, Tlalpan, Iztapalapa, Xochimilco, Milpa Alta y Tláhuac.

17 Son: Miguel Hidalgo, Benito Juárez, Cuauhtémoc y Venustiano Carranza.

18 Cárdenas Gracia, Jaime F., "Reflexiones sobre la Reforma Política al Distrito Federal", Metrópolis, Madrid, Alianza Editorial, 1992, p. 30.

19 Gamboa Montejano, Claudia, Reforma Politica del Distrito Federal, México, Cámara de Diputados, 2007, p. 25. 
Esta revista forma parte del acervo de la Biblioteca Jurídica Virtual del Instituto de Investigaciones Jurídicas de la UNAM

\section{INSTITUCIONES VIGENTES}

\section{La forma de Estado}

La Constitución Política de los Estados Unidos Mexicanos de 1917 establece el sistema federal y reconoce la soberanía de los estados para su régimen interior en su artículo 40. Además, se reconocen los poderes de la unión y los de los estados, reconociendo las Constituciones particulares de los estados para su organización respectiva. El sistema de competencias se desprende de la fórmula de las facultades expresas a las autoridades federales y las no previstas a los estados, señalada en el artículo 124 constitucional.

En relación con la capital de la nación, se incluye dentro de las partes integrantes de la Federación al Distrito Federal en el artículo 43. Y se establece a la Ciudad de México como el Distrito Federal y sede de los poderes de la Unión, considerando que en caso de que los poderes se trasladen a otro lugar, se erigirá en el Estado del Valle de México. ${ }^{20}$

Artículo que se complementa con el 122 que señala que el Distrito Federal estará a cargo de los poderes federales y de los órganos Ejecutivo, denominado jefe de gobierno del Distrito Federal, Legislativo llamado Asamblea Legislativa y Judicial representado por el Tribunal Superior de Justicia; estableciendo en el mismo artículo las bases de cada uno de dichos poderes.

Por lo cual se puede apreciar que existen dos regímenes jurídicos diferentes, uno el de los estados de la República, con reglas homogéneas para todos en el sentido de su forma de gobierno y reparto de competencias; y otro particular y único para la Ciudad de México como Distrito Federal, con un régimen especial, establecido en un Estatuto de Gobierno, el cual es promulgado por el Congreso de la Unión para reglamentar el artículo 122 constitucional ya citado.

En caso de querer trasladar o cambiar al DF, ante la propuesta de crear el Estado del Valle de México, aunque existe el procedimiento de arreglo de límites con los estados y aprobación del Senado previsto en el artículo

20 “Artículo 44. La Ciudad de México es el Distrito Federal, sede de los poderes de la Unión y capital de los Estados Unidos Mexicanos. Se compondrá del territorio que actualmente tiene y en el caso de que los poderes federales se trasladen a otro lugar, se erigirá en el Estado del Valle de México con los límites y extensión que le asigne el Congreso General" (reformado mediante decreto publicado en el Diario Oficial de la Federación, 25 de octubre de 1993). 
Esta revista forma parte del acervo de la Biblioteca Jurídica Virtual del Instituto de Investigaciones Jurídicas de la UNAM

46, sería necesaria la reforma constitucional ya que el artículo 45 señala en forma puntual que "los estados conservarán la extensión y límites que hasta hoy han tenido". Además de incluir en la lista de estados al del Valle de México en el artículo 43 que establece las partes de la Federación. Por tanto, la reforma política de crear a la Ciudad de México en entidad federativa, tiene que pasar por el proceso político de la reforma constitucional, con los inconvenientes políticos, administrativos y territoriales que esto conlleva en relación con los poderes federales y entidades vecinas involucradas.

\section{Régimen fiscal}

En primera instancia, la Ciudad de México tiene una posición de privilegio al no contar con municipios. De esta forma, todos los ingresos que no son exclusivos de la Federación pertenecen al ámbito del Gobierno de la Ciudad (por ejemplo, el predial que corresponde a los municipios conforme al artículo 115 constitucional). En segundo plano, en materia de coordinación fiscal, el Distrito Federal cuenta con una posición diferenciada del resto de los estados. Ya que en algunos casos presenta fondos y reglas especiales para la asignación de recursos. Para su análisis se procederá conforme a la propia Ley de Coordinación Fiscal, la cual presenta primero las participaciones libres y, posteriormente, las reglas de las aportaciones a las entidades federativas.

En la categoría de los recursos libres o participaciones, participa en forma igualitaria que el resto de los estados. Se destaca que la Ley en comento le da el tratamiento de "demarcaciones territoriales" en el caso del Distrito Federal para equiparar estas jurisdicciones (las actuales delegaciones) a los municipios de los estados en los fondos respectivos. Con lo cual hasta aquí no hay diferencias.

En el caso de las aportaciones existen ciertas diferencias. Respecto del Fondo para la Educación Básica y Normal, así como el de Educación Tecnológica y de Adultos, el Distrito Federal no participa con el resto de los de los estados. Estos fondos son para apoyar los servicios de educación básica y normal, principalmente en lo que corresponde al pago de maestros. El Distrito Federal no participa en razón de que dichos servicios son proporcionados directamente por la Secretaría de Educación Pública nacional (SEP), por medio del financiamiento del Ramo 25 del Presupuesto de Egresos. A partir 
Esta revista forma parte del acervo de la Biblioteca Jurídica Virtual del Instituto de Investigaciones Jurídicas de la UNAM

de 2005, la educación se imparte por la Administración Federal de Servicios Educativos, siendo un órgano desconcentrado de la SEP. ${ }^{21}$

En el caso del Fondo de Servicios de Salud no se presentan diferencias respecto de los estados al apoyar a los servicios de salud estatales que no comprenden el IMSS e ISSSTE. Sin embargo, el Fondo para la Infraestructura Social, el cual se divide en Social Estatal y Social Municipal, no participa el Distrito Federal. El Fondo de Aportaciones para el Fortalecimiento de los Municipios y Demarcaciones del D. F., aparentemente tiene un trato igual. Sin embargo, se establece $2.35 \%$ de la recaudación federal participable para todos los municipios y un $0.2123 \%$ exclusivo para las demarcaciones del DF.

El de Aportaciones Múltiples, para desayunos escolares y asistencia social a población en pobreza, corresponde a los estados y D. F. Sin embargo, se establece que se dividirá conforme a las reglas del Presupuesto de Egresos de la Federación, en el cual se ha otorgado un trato distintivo al D. F. Mismo caso se presenta en el Fondo para la Seguridad Pública en cuanto a su asignación en el Presupuesto. Y el Fondo para el Fortalecimiento de las Entidades Federativas (tanto estados como D. F.) se reparte por fórmula sin dar un trato especial a ninguna jurisdicción como tal.

Como se puede apreciar, existen algunas diferencias en cuanto al tratamiento de los estados y del Distrito Federal, como resultado no sólo del régimen constitucional del D. F., sino también de sus condiciones propias. En caso de que el D. F. se convirtiera en estado, entonces en automático dichas diferencias deberían desaparecer, como el caso de contar con un Ramo especial en el Presupuesto de Egresos federal, y darle un trato como un estado más dentro de los fondos y reglas de asignación de fondos. En esos casos, al participar como uno más, la consecuencia sería que estaría en lo que se llama en un juego en el cual de lo que ganan unos pierden otros. En el caso actual, en los casos de las diferencias ya apuntadas, el D. F. no tiene competencia alguna por dichos fondos y goza de una posición de privilegio sobre las demás entidades federativas. ${ }^{22}$

21 Auditoría Superior de la Federación, Gasto Federalizado, México, 2013, pp. 1 y 2, disponible en: http://www.asf.gob.mx/Trans/Informes/IR2009i/Tomos/Tomo5/01_ FAEB_a.pdf

22 Se anuncia la inclusión en el FAIS y la creación de un fondo especial para la Ciudad de México que "tendrá la denominación, las reglas de operación y el monto que habrá que determinar la Cámara de Diputados", como parte de las negociaciones para el presupuesto 2014 entre las autoridades federales y de la Ciudad de México. Cardoso, 
Esta revista forma parte del acervo de la Biblioteca Jurídica Virtual del Instituto de Investigaciones Jurídicas de la UNAM

\section{DeSARRollo URBANO}

Con relación al desarrollo urbano también se encuentran diferencias sustanciales entre las entidades federativas y el gobierno del Distrito Federal, pues al contar con sus propias reglas constitucionales, tiene mayor jerarquía frente a sus gobiernos locales o delegaciones. Este breve apartado presenta a continuación una síntesis sobre la problemática en cuanto a desarrollo urbano, el marco institucional en cada uno de los casos y una breve reflexión sobre las diferencias encontradas.

Es un hecho incuestionable que la principal problemática en materia urbana ha sido la dispersión y baja densidad de las ciudades en México. El $77 \%$ de la población (alrededor de 86 millones) en México es urbana y se calcula que para 2030 la proporción subirá a un $81 \%$ (Conapo, 2007). Las urbes de México generan el $86.5 \%$ del PIB; ${ }^{23}$ pero son responsables de la concentración de la pobreza y la desigualdad social, ya que alrededor del $40 \%$ de la población urbana vive en condiciones de pobreza. ${ }^{24}$ Los más pobres están obligados a vivir en la periferia urbana o zonas de riesgo, carecen de acceso a los servicios básicos y la mayoría en condiciones de irregularidad de la propiedad de la tierra que ocupan. ${ }^{25}$

Este resultado se debe en parte al modelo actual de crecimiento urbano que ha sido determinado por el mercado inmobiliario, siendo disperso, desordenado, con bajas densidades, sin usos de suelo mixto e insustentable. La construcción masiva de vivienda se ha disparado, ocasionando dicha problemática. Por ejemplo, el Infonavit otorgó poco más de 3.2 millones de créditos más que en comparación con los 30 años anteriores. ${ }^{26}$ Para 2010, el

Víctor, "Contarán DF y estados en 2014 con mayores recursos: Videgaray”, La Jornada, México, 16 de octubre de 2013, disponible en: http://www.jornada.unam.mx/2013/10/16/ capital/039n1cap

23 CIDOC-SHF, Estado actual de la vivienda en México 2012, México, Centro de Investigación y Documentación de la Casa y Sociedad Hipotecaria Federal, 2012.

24 Secretaría de Desarrollo Social, Diagnóstico del Programa Hábitat, México, 2009, disponible en: http://www.sedesol2009.sedesol.gob.mx/archivos/802567/file/Diagnostico_Habitat.pdf

25 Iracheta, Alfonso, Crisis territorial en México: la hora de la reforma urbana, México, Red de Investigaciones sobre Áreas Metropolitanas de Europa y América Latina, 2012.

26 Instituto Mexicano para la Competitividad, Viviendas para desarrollar ciudades. Índice de Competitividad en Materia de Vivienda 2011, México, 2012. 
Esta revista forma parte del acervo de la Biblioteca Jurídica Virtual del Instituto de Investigaciones Jurídicas de la UNAM

$26 \%$ de las viviendas financiadas por esta vía se encontraban desocupadas o abandonadas; siendo el $21 \%$ de los casos el resultado de la distancia que separa las viviendas de la ciudad. ${ }^{27}$

En un contexto histórico, Gutiérrez Chaparro señala que debido a la influencia de los modelos de desarrollo social y económico, junto con el intenso proceso de elaboración de planes en todo el país provocó un retroceso en la concepción, desarrollo y consolidación de la planeación urbana. Situación que se hace evidente al considerar que la planeación urbana fue promovida como una herramienta auxiliar de la política económica y social, en vez de una disciplina integral de análisis y gestión de alternativas para la ciudad. ${ }^{28}$

Esta problemática no puede compararse en forma puntual entre la Ciudad de México y el resto de las ciudades por las características históricas, políticas y económicas. Además de la evidente concentración de población y su carácter metropolitano. El D. F. cuenta con 8.8 millones de habitantes, pero al agregar los municipios contiguos del Estado de México, la población metropolitana de la Ciudad se eleva a 20.1 millones de habitantes; ${ }^{29}$ lo que ocasiona que sea el Estado de México el más poblado del país (15.1 millones de habitantes, de los cuales 12 millones aproximadamente pertenecen a la conurbación con la Ciudad de México). La densidad de población en el D. F. es de 5,920 hab/km, ${ }^{2}$ seguido del Estado de México con 679 hab/ $\mathrm{km}^{2}$, con un promedio nacional de $57 \mathrm{hab} / \mathrm{km}^{2}{ }^{30}$

Asimismo, las diferencias tampoco podrían culparse íntegramente al modelo institucional que existe entre la Ciudad de México y el resto de las ciudades y zonas metropolitanas. Sin embargo, los efectos del arreglo actual son visibles y las consecuencias notarias en relación con la política de desarrollo urbano vigente. Iniciemos por el modelo de las entidades federativas.

27 Instituto del Fondo Nacional de la Vivienda de los Trabajadores, Plan Financiero 2011-2015, México, 2010.

28 Gutiérrez Chaparro, Juan José, "Planeación urbana en México: un análisis crítico sobre su proceso de evolución”, Revista Asuntos Urbanos Internacionales, Concepción, núm. 19, 2009, p. 61.

29 Consejo Nacional de Población, Delimitación de las zonas metropolitanas de México, México, 2010, disponible en: http://www.conapo.gob.mx/es/CONAPO/Zonas_metropolitanas_2010.

30 Instituto Nacional de Estadística y Geografía, Censo de Población y Vivienda 2010, Aguascalientes, 2010, disponible en: http://www.ciberhabitat.gob.mx/impresion/ poblacion/densidad.asp. 
Esta revista forma parte del acervo de la Biblioteca Jurídica Virtual del Instituto de Investigaciones Jurídicas de la UNAM

En primer lugar, conforme a la fracción V, del artículo 115 de la Constitución, encontramos que le corresponde al gobierno municipal las competencias en materia de desarrollo urbano, las cuales comprenden la elaboración de los planes en la materia, creación de reservas territoriales, participar en los planes urbanos regionales, regular la tenencia de la tierra urbana, permisos de construcciones, reservas ecológicas y transporte público.

En segunda instancia, y derivado de la fracción XXXIX-C del artículo 73 de la Constitución, el Congreso de la Unión expidió la Ley General de Asentamientos Humanos, en la cual las competencias de la materia en estudio se encuentran divididas en forma concurrente entre los tres niveles de gobierno. Por tanto, los planes municipales de desarrollo urbano no son autónomos, sino que se encuentran subordinados a otros planes de los otros niveles de gobierno. De esta forma, existe el Plan Nacional de Desarrollo, seguido del Plan Estatal de Desarrollo Urbano. Asimismo, pueden existir planes regionales entre estados, como los casos de las zonas metropolitanas entre dos o más entidades. Además, pueden existir planes regionales a nivel estatal y metropolitanos. Por último, estarían los planes municipales, de ciudades y/o delegaciones que son las jurisdicciones menores en los municipios. Situación que se aprecia como ejemplo en el Código Urbano del estado de Querétaro. Lista y jerarquía que demuestra que si un plan municipal contiene elementos o disposiciones contrarias a los planes de niveles superiores, tales disposiciones serán nulas en consecuencia. Esta situación institucional demuestra que en las entidades federativas y sus municipios, existe un alto nivel de complejidad tanto en la planeación como en la propia administración urbana, que ocasiona un sinnúmero de conflictos tanto entre autoridades como en las autoridades frente a particulares.

En el caso del Distrito Federal el arreglo institucional no presenta mayor complejidad en razón del predominio del gobierno central frente a las delegaciones. Ya se estableció que las reglas federales no aplican. Por tanto, la competencia primaria en desarrollo urbano, que corresponde a las entidades federativas y a sus municipios, corresponde en forma total al gobierno de la Ciudad de México y no a las delegaciones. Las cuales, como ya se apuntó, no tienen competencias originarias sino delegadas del gobierno central. Y aunque existan los planes de desarrollo urbano por delegación, es incuestionable la jerarquía que tenga un plan de desarrollo urbano emanado del gobierno central, tanto en usos de suelo, política de asentamientos humanos, 
Esta revista forma parte del acervo de la Biblioteca Jurídica Virtual del Instituto de Investigaciones Jurídicas de la UNAM

movilidad y demás materias. Los planes delegaciones son complementos de los centrales y en ningún caso podrían competir frente a los centrales.

Situación que se aprecia con claridad en la Ley de Desarrollo Urbano del Distrito Federal. En principio, el artículo 6o. otorga atribuciones de primer orden al jefe de gobierno las cuales comprenden la planeación, organización y administración en la materia en estudio, así como las obras o programas que tengan un impacto en toda la Ciudad, en dos o más delegaciones o cuando así lo determine. Además, el artículo 7o. da mayor jerarquía al gobierno central cuando confiere a la Secretaría de Desarrollo Urbano y Vivienda, mayor predominio frente a las delegaciones tanto en la planeación como en la ejecución de obras y programas. ${ }^{31}$ Con estas competencias es posible asegurar la uniformidad de los planes, la regulación de los usos de suelo y todo tipo de criterios que impactan en la materia y evitar así las diferencias que son comunes en el modelo de estado con sus municipios.

Las políticas recientes que hemos visto, como el mejoramiento urbano del Centro Histórico, Polanco, Reforma, Chapultepec, las políticas de movilidad urbana (segundos pisos, metro, bici vías, etcétera), han resultado con mayor facilidad de implementación en razón de que no existe la pugna jurídica o institucional de gobierno central frente a delegaciones, o entre las mismas delegaciones. En cambio, dichos proyectos o similares, en un entorno municipal resultan de mayor dificultad en su aplicación. En principio hubieran requerido la alineación completa de los planes municipales de desarrollo de las jurisdicciones que intervinieran; además de coincidir con los del gobierno estatal. Después, el problema de la ejecución material de la obra, pasando por cuestiones presupuestarias, hasta la fase final de la administración en aquellos casos que se requiere de infraestructura.

31 Artículo 7o. Son atribuciones de la Secretaría, además de las que le confiere la Ley Orgánica, las siguientes: II. Realizar con el apoyo de las delegaciones, los estudios para la elaboración de los proyectos de programas y de sus modificaciones, para consideración del jefe de gobierno, cuidando su congruencia con los sistemas nacional y local de desarrollo; V. Revisar los proyectos de programas delegacionales y de los programas parciales, así como los de sus modificaciones, para que guarden congruencia con el Programa General de Desarrollo Urbano; VI. Supervisar los actos administrativos de las delegaciones, para vigilar el cumplimiento de los programas y de las determinaciones que corresponde emitir al jefe de gobierno en esa materia, formulando las resoluciones necesarias, así como revisar periódicamente el registro delegacional de manifestaciones de construcción...", México, Secretaría de Desarrollo Urbano y Vivienda, 2015, disponible en: http://www.seduvi.df.gob.mx/portal/index.php. 
Esta revista forma parte del acervo de la Biblioteca Jurídica Virtual del Instituto de Investigaciones Jurídicas de la UNAM

Claramente la situación actual del Distrito Federal presenta mayores ventajas en comparación con un modelo de municipios. Tiene mayores ventajas por su centralización incuestionable, mayor flexibilidad y, particularmente, cuando hay diferencias entre los planes y programas del gobierno central frente a los delegacionales, no existe controversia por la jerarquía indiscutible de los centrales. En esta materia, los municipios en México han presentado una serie de deficiencias ya conocidas, las cuales se reducen drásticamente en la Ciudad de México, por carecer competencias constitucionales sus delegaciones.

\section{PROPUESTA DE REFORMA POLÍTICA}

\section{Antecedentes}

La propuesta de reforma al gobierno de la Ciudad de México ha sido una constante desde el triunfo de Cuauhtémoc Cárdenas, del Partido de la Revolución Democrática (de izquierda) en 1997. Sin embargo, las propuestas más recientes provienen de la firma del Pacto por México del 2 de diciembre del 2012, en la cual participaron el presidente de la República y los presidentes nacionales de los principales partidos políticos. Siendo la reforma al Distrito Federal uno de los temas rectores dentro del Pacto. ${ }^{32}$

En ese orden de ideas, en octubre de 2014 el actual jefe de gobierno capitalino, Miguel Ángel Mancera Espinosa, entregó al Consejo Rector del Pacto por México su propuesta de reforma, en la cual se modificarían nueve artículos constitucionales y se asumiría como capital del país autónoma. ${ }^{33} \mathrm{Se}$ destaca el cambio en el artículo 122, en el cual se expone que la Ciudad de México continúe como capital y sede de los poderes de la Unión, además de contar con "autonomía en todo lo concerniente a su régimen interior y a su organización política y administrativa". Asimismo, se cambia el nombre de la Asamblea por el de Poder Legislativo de la Ciudad de México, el cual tendría la facultad exclusiva de aprobar adiciones o reformas a la Constitución de la Ciudad. Las delegaciones seguirían con un titular, pero

32 La Jornada, México, 3 de diciembre de 2012, disponible en: http://www.jornada. unam.mx/2012/12/03/politica/002n1pol.

33 El Financiero, México, 2 de octubre de 2014, disponible en: http://www.elfinanciero.com.mx/politica/mancera-entrega-a-pacto-por-mexico-propuesta-de-reforma-df.html. 
se agrega la figura de un órgano colegiado de elección popular directa que tendría facultades para supervisar y evaluar al gobierno y gasto público.

En síntesis, se propone definir el nombre oficial de la Ciudad de México; dotar de una Constitución a la Ciudad; revisar las competencias del jefe de gobierno y de la Asamblea; y fortalecer a las delegaciones creando órganos colegiados (tipo ayuntamientos). Como se puede apreciar, aunque se reconoce ya la dificultad de crear un nuevo estado de la República, se optó por la vía de la "autonomía" pero equiparando las figuras políticas y administrativas a la organización que cuentan los estados. En primera instancia es necesario definir cuál es la finalidad de la reforma para de ahí construir el esquema constitucional ideal. ¿Se busca sólo fortalecer las competencias y figuras de los poderes Ejecutivo y Legislativo de la Ciudad? ¿Se busca dotar de mayores facultades políticas a los ciudadanos? O ¿Se busca eficientar los servicios públicos? Con estas ideas se procede al análisis de las propuestas e iniciativas, por ser las más importantes dentro del proceso de reforma constitucional y, posteriormente, en el capítulo de conclusiones se retoman junto a la última reforma constitucional.

\section{Nombre oficial}

En relación con dotar a la Ciudad de México de un nombre oficial en el lenguaje constitucional, el señalar un nombre o concepto a cierta demarcación territorial con jurisdicción es contar con un nombre oficial. De tal forma, el artículo 43 constitucional define los nombres oficiales de los estados de la República. Y el 44 establece puntualmente que "la Ciudad de México es el Distrito Federal". Por tanto, si existe un nombre oficial que es "La Ciudad de México" o "Gobierno de la Ciudad de México". En todo caso, si se busca modificar el nombre se debe contemplar la reforma a los artículos 43, 44 y 122 entre otros, para que no haya duda en cuanto al nombre que deba contar el Distrito Federal.

\section{Constitución local}

La propuesta de reforma contempla conservar la condición de autonomía o trato especial que existe hoy en día. Bajo estas líneas, sería forzoso en primer término que el artículo 122 constitucional establezca 
Esta revista forma parte del acervo de la Biblioteca Jurídica Virtual del Instituto de Investigaciones Jurídicas de la UNAM

en forma puntual la autonomía de la Ciudad de México respecto de su forma de gobierno y organización política interna. Asimismo, dada la importancia que tiene el Distrito, es indispensable que dicho artículo siga estableciendo la organización de los poderes y competencias mínimas; de ahí que el siguiente documento legal deba reglamentar tales principios constitucionales.

Ahora bien, respecto de la técnica constitucional en materia de autonomías, existen dos formas distintas para reglamentar el texto fundamental. En el primer caso se otorga la autonomía y, al mismo tiempo, se concede la competencia amplia para que la "entidad" en cuestión se dé su propia normatividad sin existir un estatuto o ley reglamentaria por parte del legislativo nacional. En estos casos se trata de situaciones de pueblos o grupos minoritarios en los cuales se les concede dicha libertad para regular sus usos y costumbres por su condición especial.

En el segundo caso, después del reconocimiento constitucional de autonomía es necesario que el Legislativo o parlamento nacional promulgue un estatuto de autonomía en el cual se establezcan las competencias y la organización de los poderes básicos de la nueva jurisdicción; con lo cual queda vedada la posibilidad de una Constitución propia de la "entidad" por lo menos en el sentido formal de contar con una entidad que cuenta con soberanía interior para dicho fin. Este segundo caso es propio de un ámbito territorial mayor y con pueblos o naciones distintivas. Dicho estatuto podrá ser reglamentado por la "entidad", pero ya no se tratará de una Constitución sino de una ley reglamentaria del estatuto. ${ }^{34}$

Desde esta perspectiva, la situación del Distrito Federal claramente no responde a la primera situación que es propia de grupos en minoría. Sino al segundo caso de ámbito territorial, si bien no es una nación o pueblo diferente, sí es un grupo de población con características especiales por ser el asiento de los poderes de la Unión. Por tanto, no podría existir una Constitución en el sentido de las Constituciones de los estados, sino una ley secundaria y reglamentaria del Estatuto de Gobierno emanada del Congreso de la Unión. Si lo que se busca es que dicho cuerpo normativo secundario sea expedido por un órgano y procedimiento especial, diferente al de la creación de leyes ordinarias, no existiría ningún problema constitucional en tanto dicho

34 Muñoz Machado, Santiago, "El mito del Estatuto-Constitución y las reformas estatutarias, Informe Comunidades Autónomas", Informe Comunidades Autónomas 2004, Barcelona, Instituto de Derecho Público, 2004, pp. 735-756. 
Esta revista forma parte del acervo de la Biblioteca Jurídica Virtual del Instituto de Investigaciones Jurídicas de la UNAM

cuerpo normativo, sin importar su nombre, quede subordinado al Estatuto de Gobierno. Siendo la razón de este análisis la falta de soberanía interior por tratarse precisamente de una zona o entidad con autonomía.

\section{Competencias del Ejecutivo y el Legislativo}

En cuanto a las facultades del Ejecutivo y competencias de la Asamblea o nuevo Legislativo de la Ciudad, esto en primer término sería en razón directa del catálogo de competencias que se reconozcan en el artículo 122 constitucional.

De acuerdo con los referentes constitucionales en materia de autonomía, se pueden citar ejemplos de plena autonomía (en materia de seguridad pública y recaudación impositiva) hasta autonomías muy acotadas. La respuesta no puede proceder de la mera técnica constitucional sino de factores políticos, económicos y sociales de la capacidad de la Ciudad de México para contar con competencias propias para brindar servicios y programas. Por sus dimensiones socioeconómicas y condiciones de urbanismo, podríamos señalar que podrían ser mayores o "especiales" a las que tienen los gobiernos estatales. Sin embargo, la cuestión se complica un poco más por ser sede de los poderes federales y, en esa situación, no sería posible otorgar una autonomía muy amplia porque iría en contra de asegurar un campo de acción a los poderes de la Unión. Un ejemplo es el caso muy notorio en materia de seguridad y gobernabilidad, en el cual el mando tiene que estar bajo el presidente de la República. Por lo tanto, una buena medida sería iniciar por aquellas competencias necesarias o indispensables que tienen que estar forzosamente dentro del ámbito federal para asegurar la protección y buen funcionamiento de los poderes de la Unión.

\section{Delegaciones}

La reforma en el sentido de equiparar las delegaciones a los municipios y que cuenten con órganos colegiados similares a los ayuntamientos nos remite también al problema de cuáles son los servicios básicos que darían las delegaciones por su propia cuenta. Si no existe un catálogo de servicios básicos a este nivel, no tiene sentido contar con un órgano de representa- 
Esta revista forma parte del acervo de la Biblioteca Jurídica Virtual del Instituto de Investigaciones Jurídicas de la UNAM

ción política que sólo va a dar opiniones pero sin autoridad alguna para las cuestiones públicas.

Esto viene en razón de que desde los siglos XIX y XX, el gobierno central de la Ciudad de México en múltiples ocasiones quitó y concentró servicios en razón de las deficiencias y grandes diferencias entre los municipios y ahora entre las delegaciones. Es importante analizar si se van a crear "entes" con personalidad jurídica y presupuestos propios para hacerse responsables de servicios básicos a la ciudadanía. Si tal fuera el caso, una fórmula en este sentido podría ser la creación de un catálogo de servicios mínimos para todas las delegaciones y funcionar por medio de convenios con la autoridad central de la Ciudad en los demás servicios, para que así cada delegación pudiera contar con un traje a la medida de sus propias necesidades. Por otro lado, esta reforma podría ser posible hoy en día mediante la reforma del actual Estatuto de la Ciudad de México, sin la necesidad de realizar una reforma constitucional.

\section{CONCLUSIONES}

\section{Problemática}

La trayectoria histórica de la Ciudad de México se encuentra marcada por los distintos modelos constitucionales del siglo XIX. Sin embargo, en cualquiera de ellos el gobierno se organizó en torno a una figura administrativa central y municipios. Ya en el siglo XX se suprime el municipio y aparecen las delegaciones actuales. La creación de la Asamblea de Representantes en 1987 así como la reforma política de 1993 y 2016, que establece un régimen especial de gobierno de la Ciudad, representan hechos fundamentales en su forma de Estado.

En particular la reforma política en los últimos años osciló entre crear un estado más de la República hasta propuestas para ampliar su autonomía. El Pacto por México encontró retrasos ante el desgaste político por la aprobación y ejecución de las reformas estructurales; el proceso electoral intermedio (2015) respecto al sexenio presidencial (2012-2018); así como la división de la izquierda en dos partidos al separarse Andrés Manuel López Obrador (ex candidato presidencial y ex jefe de gobierno del D. F.) del Partido de la Revolución Democrática y fundar un nuevo partido (Movimiento 
Esta revista forma parte del acervo de la Biblioteca Jurídica Virtual del Instituto de Investigaciones Jurídicas de la UNAM

Regeneración Nacional), quedando las delegaciones y Asamblea del D. F. divididas entre estos dos partidos.

Sin embargo, el 29 de enero de 2016, se publicó en el Diario Oficial, la reforma constitucional que modificó varios artículos para crear el gobierno de la Ciudad de México. Siendo el artículo 122 constitucional el principal en cuestión. ${ }^{35} \mathrm{El}$ artículo se encuentra dividido en tres apartados que establecen la forma de gobierno, las reglas federales y las normas metropolitanas. Respecto de los puntos de análisis de la iniciativa del Pacto de México, caben las siguientes reflexiones:

\section{Resultados}

A. Nombre oficial y naturaleza jurídica. La reforma ha borrado el nombre de Distrito Federal quedando solamente "La Ciudad de México". Si bien este nombre oficial ya existía como lo señalamos anteriormente, técnicamente sigue siendo un distrito federal por las competencias que sigue teniendo el gobierno federal respecto de este territorio, como se verá a continuación. En primer término no se ha creado un estado más de la República ya que desde su naturaleza jurídica se anuncia un régimen especial al señalar su autonomía en su forma política. Aunque desde el inicio la redacción se presta a contradicciones ya que señala que es una entidad federativa. La única forma de salvar este inconveniente es interpretando que entidad federativa es un término genérico que hace alusión a cualquier entidad o jurisdicción que forme parte de la República y no en el sentido restrictivo de los estados que conforman la Unión. ${ }^{36}$

B. Constitución política. Si bien la reforma incluyó el nombre de Constitución para la Ciudad de México, así como un poder constituyente; en consideración de la técnica constitucional es en realidad un Estatuto de Gobierno propio de una entidad autónoma. Las Constituciones locales o estatales son promulgadas por los estados o entidades federativas que tienen soberanía. Esto es, no participan los poderes federales en su promulgación. En el presente caso, de acuerdo a la reforma, el Congreso de la Unión par-

35 Véase México, UNAM, Instituto de Investigaciones Jurídicas, 2016, disponible en: http://info4.juridicas.unam.mx/ijure/fed/9/123.htm? $s=$

36 Artículo 122. La Ciudad de México es una entidad federativa que goza de autonomía en todo lo concerniente a su régimen interior y a su organización política y administrativa. 
Esta revista forma parte del acervo de la Biblioteca Jurídica Virtual del Instituto de Investigaciones Jurídicas de la UNAM

ticipará en forma permanente en el poder constituyente de la Ciudad de México. Sin la participación del Congreso federal, eso hubiera representado una soberanía, así como la creación constitucional de un estado más de la República, con la consecuente traslación de los poderes federales, entre otros puntos ya analizados. Reconociendo que se ha creado un "Estatuto" especial en consideración de la participación del Congreso federal con los poderes propios de la Ciudad.

C. Competencias del Ejecutivo y el Legislativo. El artículo 122 constitucional en el apartado A establece la forma de gobierno y estructura orgánica de los tres poderes, órganos constitucionales y régimen paraestatal. Dejando poco margen a su "Constitución local" para crear un régimen especial o autónomo. Es de notarse que se ha creado un régimen idéntico al de los estados de la República, perdiéndose una valiosa oportunidad para crear instituciones políticas nuevas que puedan obedecer a la realidad de esta importante Ciudad.

En relación al régimen federal, el artículo 122, en su apartado B, establece la división de competencias entre la Federación y la Ciudad, así como las reglas de las autoridades federales respecto del territorio en la Ciudad de México. Respecto al primer punto el apartado B inicia: "Los poderes federales tendrán respecto de la Ciudad de México, exclusivamente las facultades que expresamente les confiere esta Constitución". Al ser la única mención respecto este punto, se entiende, mediante una interpretación gramatical, que las reglas a favor de la Federación, ya contenidas en el resto de la Constitución, se reproducen en forma idéntica respecto a la Ciudad. Por tanto, y como ejemplo, las reglas del artículo 73 constitucional aplican dentro de este "régimen especial". Lo que ocasiona que las reglas sobre federalismo, esto es, división de competencias entre Federación y estados, sean las mismas que entre la Federación y la Ciudad de México. Con ello se pierde una valiosa ventaja o privilegio que tenía la Ciudad de México, ya que al reproducir esta nueva regla deberá contar con las mismas competencias que cualquier estado; perdiéndose la esencia propia de una autonomía.

Respecto de las reglas de las autoridades federales sobre el territorio de la Ciudad, se reproducen las mismas que ya existían antes de la reforma. Lo cual confirma la situación jurídica y de hecho que continúa siendo un distrito federal con un régimen especial; como el mando del Ejecutivo federal respecto de la fuerza pública. 
D. Delegaciones. La reforma suprime las delegaciones y crea las alcaldías. Aunque se remitirá a la Constitución de la Ciudad su organización, el artículo 122 constitucional establece ya la estructura política y orgánica de las alcaldías, siendo casi idéntica al régimen municipal del artículo 115 constitucional. Aquí otra vez se ha perdido la oportunidad de contar con un "Estatuto", Constitución o régimen especial para los gobiernos locales de la Ciudad al reproducir desde la Constitución su organización política.

Sin embargo, en el tema de la división de competencias entre el gobierno de la Ciudad y las alcaldías todavía existe un margen importante para contar con un régimen local especial en cuanto a competencias, ya que éste se va a reglamentar en la Constitución local, y omitir el problema fundamental de los municipios en México, que ha consistido en que todos tengan la misma estructura y competencias sin importar sus diferencias en todos sentidos tan marcadas. Esto es, crear regímenes especiales o particulares de acuerdo con las características y circunstancias propias de cada una de las alcaldías, ya sea mediante un estatuto que contemple diferentes tipos de gobiernos locales o mediante la promulgación de estatutos particulares para cada una de las demarcaciones.

\section{Consideraciones finales}

Se estableció como hipótesis de trabajo que convertir a la Ciudad de México en estado federal le restaría competencias y privilegios. Y ampliar su autonomía le daría mayores competencias. En consideración de los resultados arriba citados, así como la reciente reforma constitucional, la hipótesis se ha probado en forma parcial. Efectivamente no se ha creado un estado más de la República, evitándose con ello los problemas ya anotados como el hecho de trasladar a los poderes federales. Sin embargo, se ha creado un régimen especial o entidad autónoma pero sólo en cuanto a la forma, en el fondo los principios constitucionales le quitaron o limitaron su condición especial.

En cuanto a la forma se prueba la condición de autonomía o régimen especial mediante la participación del Congreso federal en su Constitución local. Creando de esta manera un régimen especial y distinto al de los estados de la República. Asimismo, su reglamentación en un artículo único, esto es el 122, crea una situación especial dentro de la Constitución. 
Esta revista forma parte del acervo de la Biblioteca Jurídica Virtual del Instituto de Investigaciones Jurídicas de la UNAM

En cuanto al fondo, se han conservado las mismas competencias que ya se tenían. Inclusive pudiera ser que la Ciudad disminuya sus ventajas al introducir la regla por la cual la Federación conserva todas sus competencias establecidas en el resto de la Constitución general, respecto al artículo 122 de la carta. Perdiéndose por el momento una oportunidad única e histórica para que la Ciudad de México pudiera tener en verdad un régimen de competencias único y ajustado a su propia realidad. Quedan como puntos salvables, y en espera de una legislación secundaria que le dé vida y no repita los mismos esquemas, las competencias entre la Ciudad y las alcaldías, así como los asuntos metropolitanos.

\section{BIBLIOGRAFÍA}

Auditoría Superior de la Federación, Gasto federalizado, México, 2013, disponible en: http://www.asf.gob.mx/Trans/Informes/IR2009i/ Tomos/Tomo5/01_FAEB_a.pdf.

CÁmara de Diputados, Leyes, México, H. Congreso de la Unión, septiembre de 2013, disponible en: http://www.diputados.gob.mx/LeyesBiblio/pdf/31.pdf.

CÁRDENAS GRACIA, Jaime F., "Reflexiones sobre la Reforma Política al Distrito Federal”, Metrópolis, Madrid, Alianza, 1992.

CARDoso, Víctor, "Contarán DF y estados en 2014 con mayores recursos: Videgaray", La Jornada, México, 16 de octubre de 2013, disponible en: http://www.jornada.unam.mx/2013/10/16/capital/039n1cap.

CIDOC-SHF, Estado actual de la vivienda en México 2012, México, Centro de Investigación y Documentación de la Casa y Sociedad Hipotecaria Federal, 2012.

Consejo Nacional de Población, México, 2007, disponible en: http:// www.conapo.gob.mx/.

—- Delimitación de las zonas metropolitanas de México, México, 2010, disponible en: http://www.conapo.gob.mx/es/CONAPO/Zonas metropolitanas_2010.

Dublán, Manuel y LozAno, José María, Legislación mexicana, México, Imprenta del Comercio, 1876, t. VII. 
Esta revista forma parte del acervo de la Biblioteca Jurídica Virtual del Instituto de Investigaciones Jurídicas de la UNAM

\section{XXXV.}

Legislación mexicana, México, Imprenta del Comercio, 1876, t.

El Financiero, México, 2 de octubre de 2014, disponible en: http://www. elfinanciero.com.mx/politica/mancera-entrega-a-pacto-por-mexicopropuesta-de-reforma-df.html.

FERRANDO BADÍA, Juan, El Estado unitario, el federal y el Estado autonómico, 2a. ed., Madrid, Tecnos, 1986.

Gamboa Montejano, Claudia, Reforma Política del Distrito Federal, México, Cámara de Diputados, 2007.

GonzÁlez Casanova, J. A., Teoría del Estado y derecho constitucional, 3a. ed., Barcelona, Vicens-Vives, 1989.

GUTIÉRrez CHAPARRo, Juan José, "Planeación urbana en México: un análisis crítico sobre su proceso de evolución", Revista Asuntos Urbanos Internacionales, Concepción, núm. 19, 2009.

Instituto del Fondo NACIONAL DE LA ViviendA DE LOS TRABAJAdORES, Plan Financiero 2011-2015, México, 2010.

Instituto MEXICANO PARA LA COMPETITIVIDAD, Viviendas para desarrollar ciudades. Índice de Competitividad en Materia de Vivienda 2011, México, 2012.

Instituto Nacional de Estudios Históricos DE LA ReVolución Mexicana, Congreso Constituyente 1916 a 1917, Diario de Debates, México, INEHRM, 1985, t. II.

Instituto Nacional para el Federalismo y el Desarrollo MuniCIPAL, México, Secretaría de Gobernación, 2013, disponible en: http:// www.inafed.gob.mx/work/enciclopedia/EMM09DF/historia.html.

Instituto Nacional de Estadística y Geografía, Censo de Población y Vivienda 2010, Aguascalientes, 2010, disponible en: http://www. ciberhabitat.gob.mx/impresion/poblacion/densidad.asp.

IRACHETA, Alfonso, Crisis territorial en México: la hora de la reforma urbana, México, Red de Investigaciones sobre Áreas Metropolitanas de Europa y América Latina, 2012.

La Jornada, México, 3 de diciembre de 2012, disponible en: http://www. jornada.unam.mx/2012/12/03/politica/002n1pol.

MuÑOZ MACHADO, Santiago, "El mito del Estatuto-Constitución y las reformas estatutarias, Informe Comunidades Autónomas", Informe Co- 
Esta revista forma parte del acervo de la Biblioteca Jurídica Virtual del Instituto de Investigaciones Jurídicas de la UNAM www.juridicas.unam. $\mathrm{mx}$

munidades Autónomas 2004, Barcelona, Instituto de Derecho Público, 2004.

SAlOMÓN DÍAZ, Alfaro, "El Distrito Federal mexicano. Breve historia constitucional", Estudios jurídicos en torno a la Constitución mexicana de 1917 en su septuagésimo quinto aniversario, México, UNAM, 1992.

Secretaría de Desarrollo Social, Diagnóstico del Programa Hábitat, México, 2009, disponible en: http://www.sedesol2009.sedesol.gob. $m x /$ archivos/802567/file/Diagnostico_Habitat.pdf.

Tena RAMíREZ, Felipe, Derecho constitucional mexicano, 22a. ed., México, Porrúa, 1987.

— Leyes fundamentales de México, México, Porrúa, 1985. 